OPEN ACCESS

Edited by:

Xiongwei Zhu,

Case Western Reserve University,

United States

Reviewed by:

Pingyi $X u$,

First Affiliated Hospital of Guangzhou Medical University, China

*Correspondence:

Fang Huang

huangf@shmu.edu.cn

Yun-Cheng Wu

yunchw@medmail.com.cn;

drwu2006@163.com

Received: 21 October 2020

Accepted: 13 April 2021

Published: 06 July 2021

Citation:

Chen Y, WU T, Li H, LiX, Li Q, Zhu X,

Yu M, Kuo S-H, Huang F and Wu Y-C

(2021) Corrigendum:

DI-3-n-Butylphthalide Exerts

Dopaminergic Neuroprotection

Through Inhibition of

Neuroinflammation.

Front. Aging Neurosci. 13:620015

doi: 10.3389/fnagi.2021.620015

\section{Corrigendum: DI-3-n-Butylphthalide Exerts Dopaminergic Neuroprotection Through Inhibition of Neuroinflammation}

\author{
Yajing Chen ${ }^{1}$, Tingting $W u^{1}$, Heng $L i^{2}$, Xuan $L i^{1}$, Qing $L i^{3}$, Xiaoying Zhu ${ }^{1}$, Mei $Y u^{3}$, \\ Sheng-Han Kuo ${ }^{4}$, Fang Huang ${ }^{3 *}$ and Yun-Cheng $\mathrm{Wu}^{1 *}$
}

\begin{abstract}
${ }^{1}$ Department of Neurology, Shanghai General Hospital, Shanghai Jiao Tong University School of Medicine, Shanghai, China, ${ }^{2}$ Department of Neurology, Jinan Central Hospital Affiliated to Shandong University, Jinan, China, ${ }^{3}$ The State Key Laboratory of Medical Neurobiology, The Institutes of Brain Science and the Collaborative Innovation Center for Brain Science, Shanghai Medical College, Fudan University, Shanghai, China, ${ }^{4}$ Department of Neurology, College of Physicians and Surgeons, Columbia University, New York, NY, United States
\end{abstract}

Keywords: dl-3-n-butylphthalide, MAPK, microglia, neuroinflammation, NF-kB, Parkinson's disease

\section{A Corrigendum on}

Dl-3-n-Butylphthalide Exerts Dopaminergic Neuroprotection Through Inhibition of Neuroinflammation

by Chen, Y., Wu, T., Li, H., Li, X., Li, Q., Zhu, X., et al. (2019). Front. Aging Neurosci. 11:44. doi: 10.3389/fnagi.2019.00044

In the original article, there was a mistake in Figure 5B, Figure 6B, and Figure $\mathbf{8 B}$ as published. The image in Figure 5B (NBP) was inadvertently replaced with the image from Figure 5B (NBP+LPS). In Figure 6B, the extra bands of COX-2 were mistaken as the main bands. In Figure $8 \mathrm{~B}$, the images of cytoplasmic p65 and actin bands were inadvertently exchanged during images organization. The corrected Figure 5, Figure 6, and Figure 8 appear below.

The authors apologize for these errors and state that this does not change the scientific conclusions of the article in any way. The original article has been updated.

\footnotetext{
Copyright $\odot 2021$ Chen, $W u, L i, L i, L i, Z h u, Y u, K u o, H u a n g$ and $W u$. This is an open-access article distributed under the terms of the Creative Commons Attribution License (CC BY). The use, distribution or reproduction in other forums is permitted, provided the original author(s) and the copyright owner(s) are credited and that the original publication in this journal is cited, in accordance with accepted academic practice. No use, distribution or reproduction is permitted which does not comply with these terms.
} 

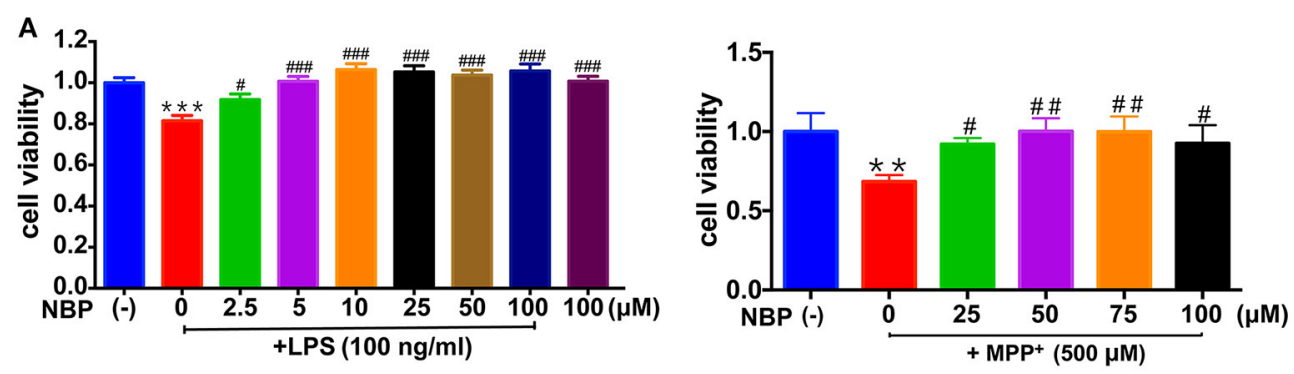

B

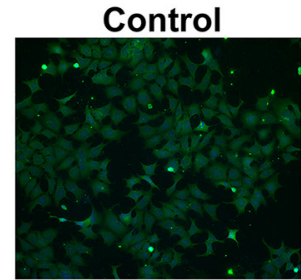

NBP

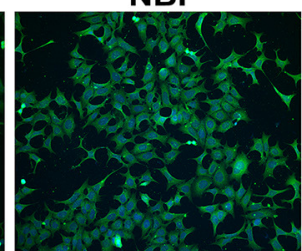

LPS

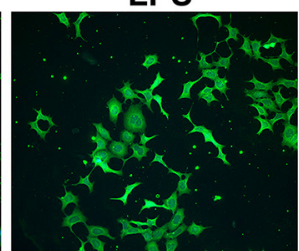

NBP+LPS

C

Control

NBP

LPS
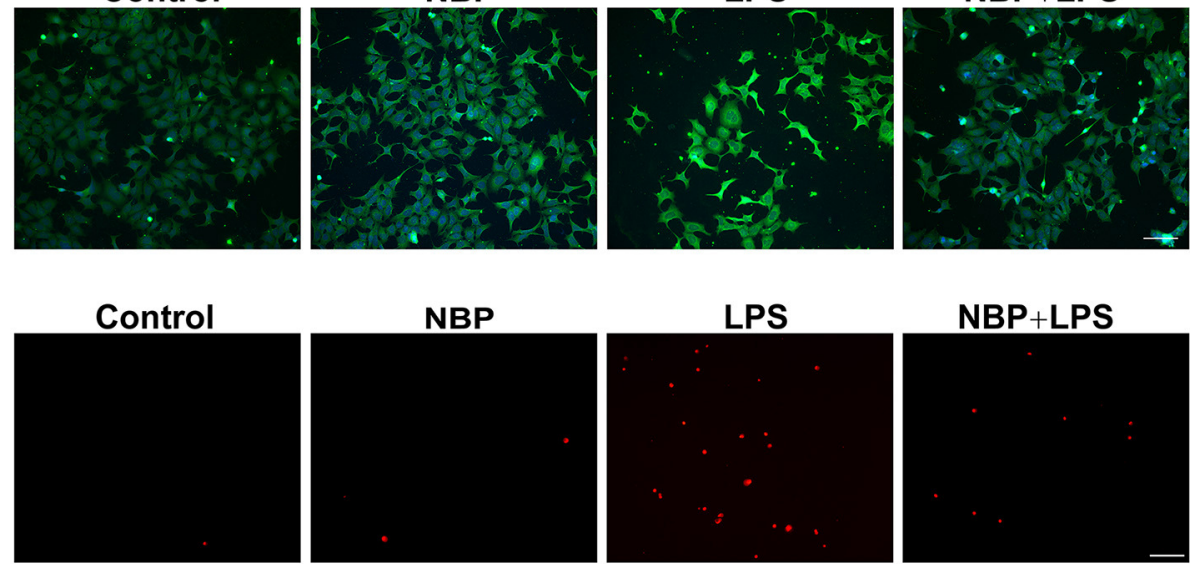

NBP+LPS

FIGURE 5 | NBP protected dopaminergic neurons from neurotoxicity induced by microglial activation. SH-SY5Y cells were incubated for $24 \mathrm{~h}$ with conditioned medium derived from cultures of BV-2 cells. Before collecting culture media, BV-2 cells were pretreated with NBP $(0$ or $100 \mu \mathrm{M})$ for $1 \mathrm{~h}$ and incubated with LPS $(0$ or $100 \mathrm{ng} / \mathrm{ml})$ or $\mathrm{MPP}^{+}(0$ or $500 \mu \mathrm{M})$ for $24 \mathrm{~h}$. (A) Cell viability was measured with the CCK8 assay $(n=5)$. All data are presented as means $\pm \mathrm{SEM}$. ${ }^{* \star} p<0.01,{ }^{* \star *} p<$ 0.001 , compared with the Control group; ${ }^{*} p<0.05$, $\# \# p<0.01$, \#\#\# $p<0.001$, compared with the LPS group or the MPP ${ }^{+}$group. (B) The apoptosis of SH-SY5Y was evaluated by immunofluorescence detection of cleaved caspase-3 (green) and cell nuclei was stained with DAPI (blue) (scale bar: $50 \mu \mathrm{m})$. (C) The cell death of SH-SY5Y was evaluated by immunofluorescence detection of PI (red) (scale bar: $50 \mu \mathrm{m}$ ). 
A
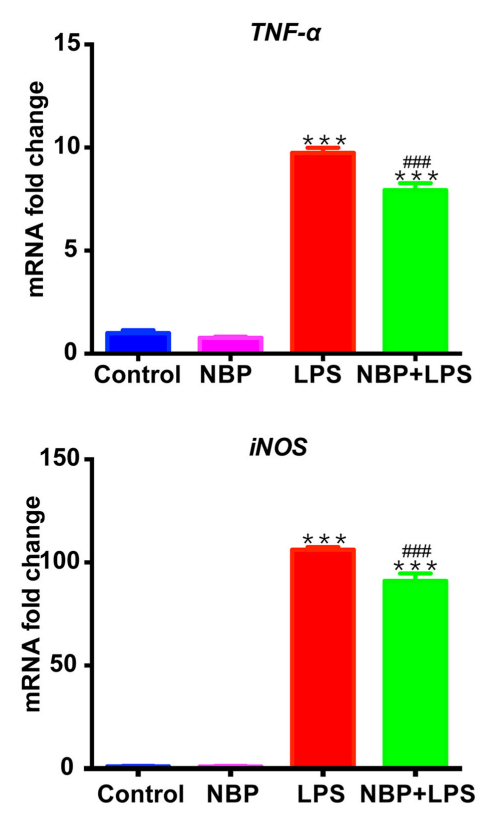

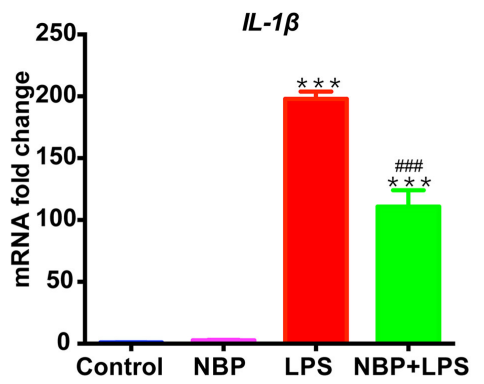

cox-2

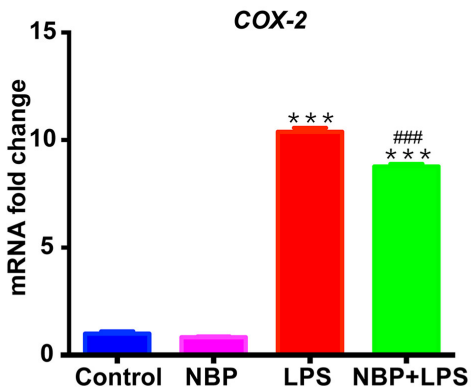

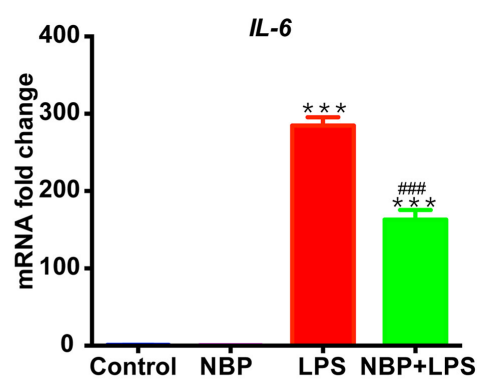

B

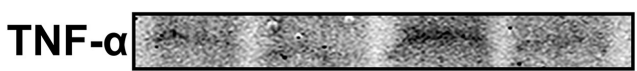

actin

IL-1 $\beta$

actin
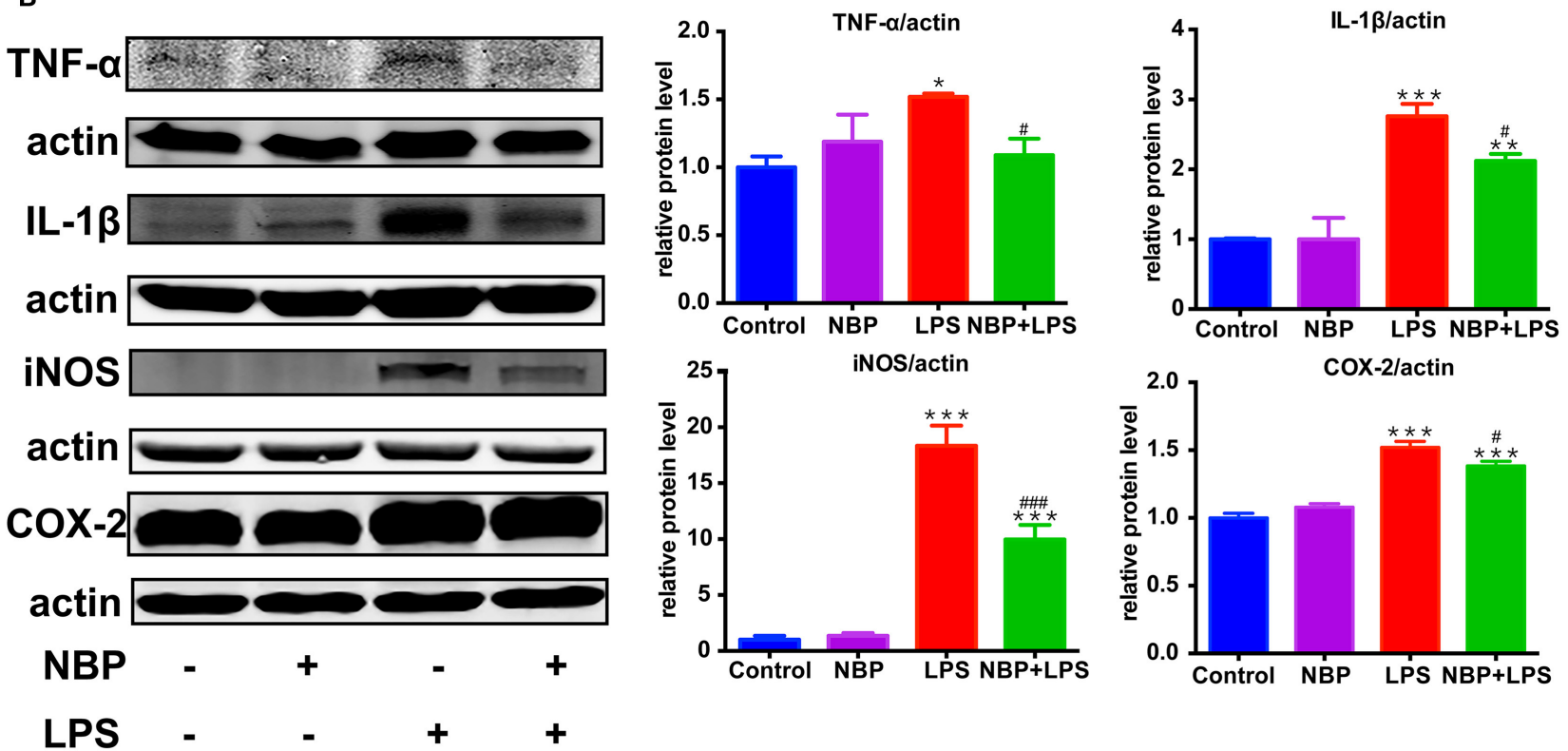

C
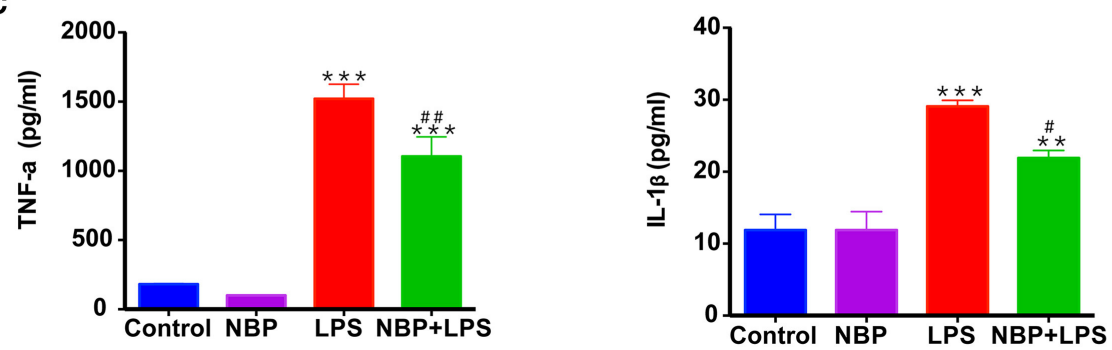

FIGURE 6 | NBP reduced pro-inflammatory molecules expression in LPS-stimulated BV-2 cells. BV-2 cells were pretreated with NBP $(0$ or $100 \mu \mathrm{M})$ for $1 \mathrm{~h}$ followed by LPS $(0$ or $100 \mathrm{ng} / \mathrm{ml})$ for $6 \mathrm{~h}$ and total RNA was isolated. To measure cellular protein expression or cytokines level in supernatants, time for LPS treatment was $24 \mathrm{~h}$. (A) The mRNA expression of IL-1 $\beta$, IL-6, TNF-a, iNOS and COX-2 was analyzed by RT-PCR and normalized to that of $\beta$-actin. (B) The protein level of TNF- $\alpha$, IL-1 $\beta$, iNOS and COX2 was analyzed by Western Blot. (C) The level of TNF- $\alpha$ and IL-1 $\beta$ in supernatants was assayed by ELISA kit. All data are presented as means \pm SEM $(n=$

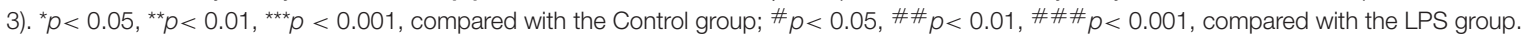


A
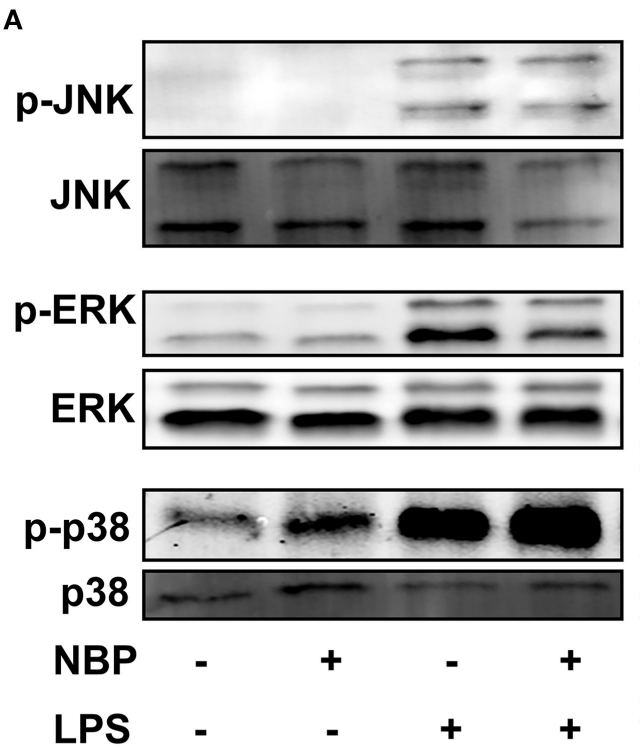

B

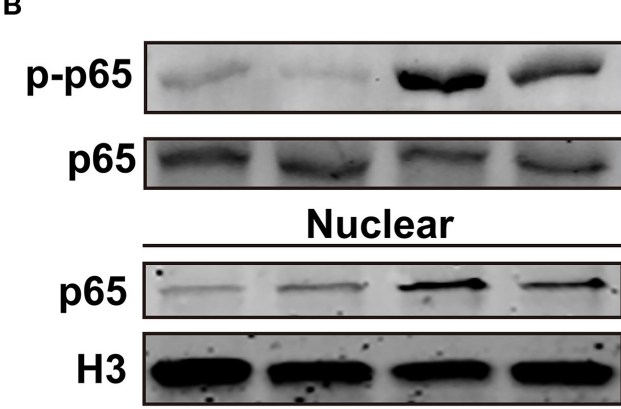

Cytoplasmic

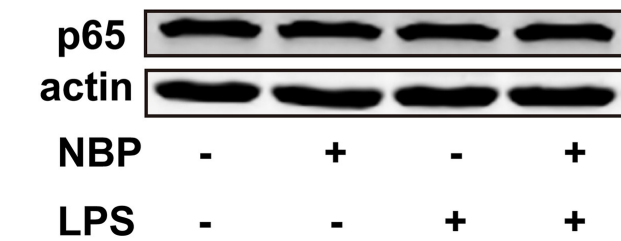

C

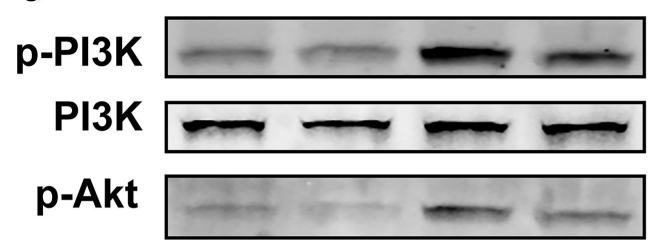

Akt
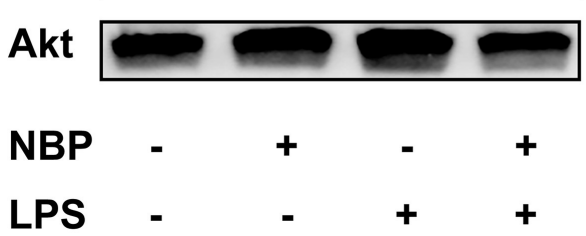
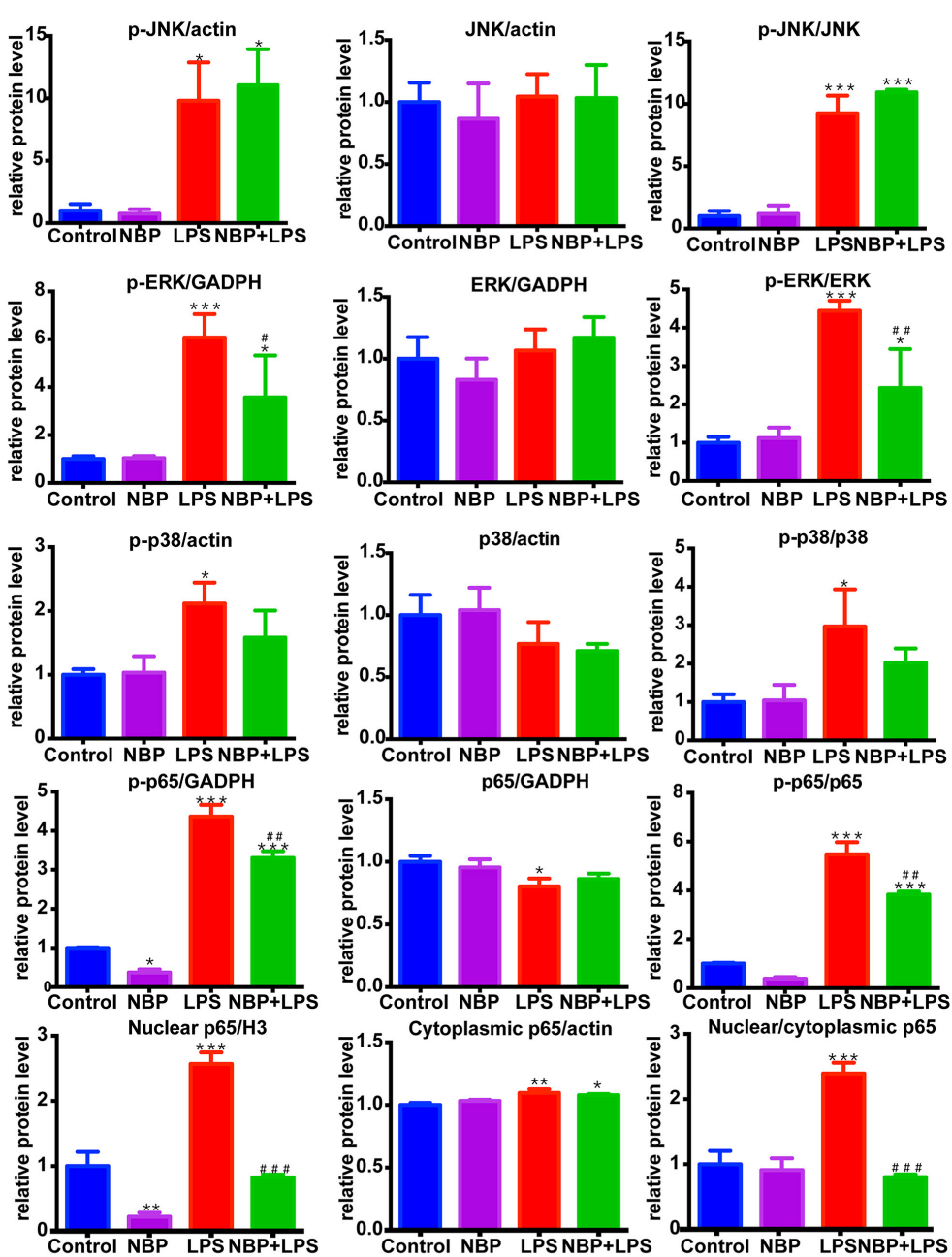

Control
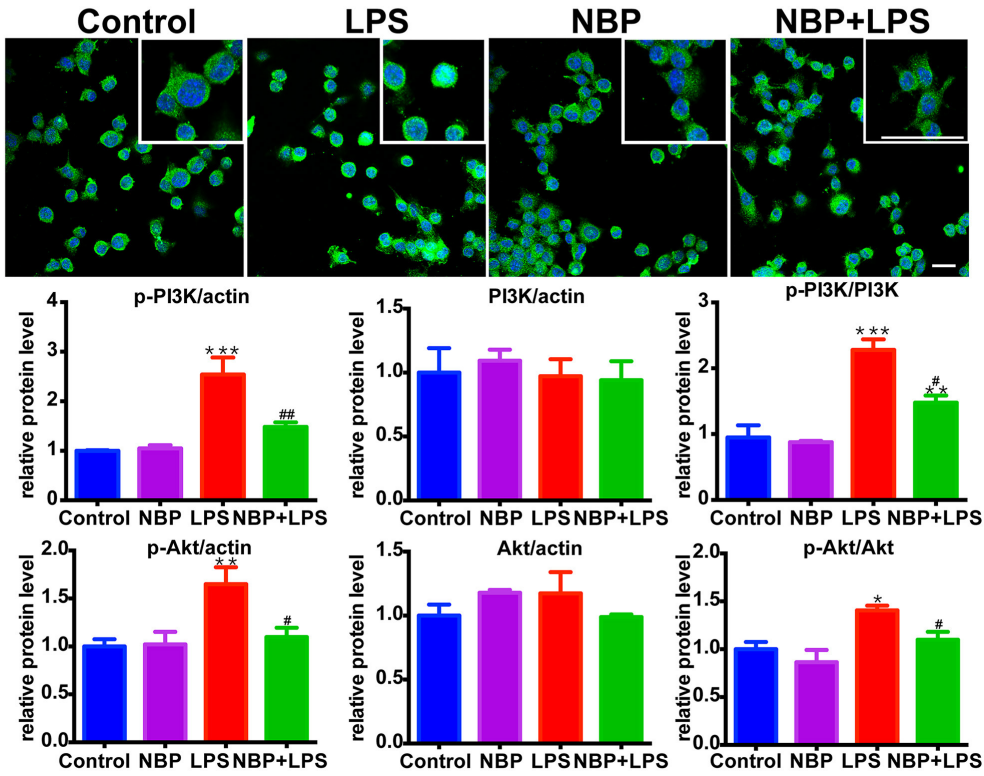

FIGURE 8 | NBP inhibited the activation of ERK, NF-kB and PI3K/Akt pathways in LPS-stimulated BV-2 cells. (A) Western Blot assay for MAPK expression. The expression of total JNK/p38/ERK as well as p-JNK/p-p38/p-ERK were analyzed. (B) Western Blot assay for NF-kB expression in whole-cell, nuclear and cytoplasmic extracts. The nuclear translocation of p65 was also evaluated by immunofluorescence detection of p65 (green) and cell nuclei was stained with DAPI (blue) (scale bar: $50 \mu \mathrm{m})$. (C) Western Blot assay for PI3K/Akt expression. The expression of total PI3K/Akt as well as p-PI3K/p-Akt were analyzed. All data are presented as means \pm

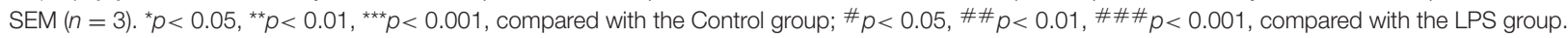

